\title{
Calculation of Eigenvalues for Eddy Current Testing Problems
}

\author{
Valentina Koliskina, Riga Technical University
}

\begin{abstract}
Semi-analytical solutions of eddy current testing problems require several computational steps. One of the steps where numerical methods are needed is calculation of complex eigenvalues without good initial approximation for the roots. In the presented paper we describe three eddy current testing problems with cylindrical symmetry where a cylindrical inclusion in a conducting medium is of finite size. In all three cases eigenvalue problem reduces to transcendental equations containing Bessel functions in a complex plane. The algorithm of the solution of such problems is described in the paper. Results of numerical computation are presented.
\end{abstract}

Keywords-Bessel functions, complex eigenvalues, eddy current testing, flaw detection, TREE method.

\section{INTRODUCTION}

Eddy current method is widely used for quality testing of electrically conducting materials. The method is based on the principle of electromagnetic induction. If a conducting medium (for example, a metal plate) is located near a source of alternating current (for example, a coil) then eddy currents are induced in the medium. These currents, in turn, interact with the currents in the coil changing the impedance of the coil. If a flaw is present in a conducting medium then the parameters of the flaw are usually estimated solving the inverse problem, where the difference (in some norm) between experimental and theoretical data is minimized [1]. Thus, a reliable and accurate method for the solution of a direct problem is needed in order to solve the inverse problem.

The classical approach for solving direct problems is based on the assumption that a conducting medium is infinite in one or two spatial dimensions. Method of integral transforms (such as Hankel of Fourier transforms) is used in such cases to compute the change in impedance of the coil [2], [3]. In many cases, however, conducting medium is of finite size. Numerical methods such as finite element methods are usually used in cases of complex geometry of the conducting medium [4]. Applications include coin validation, estimation of the effects of corrosion and analysis of other flaws in a conducting medium.

Semi-analytical solutions for problems where a conducting medium is of finite size can be constructed by the TREE method [3], [5]. The basic assumption in the TREE method is that the electromagnetic field is assumed to be exactly zero at a sufficiently large distance from the coil. In this case the boundary conditions of the first or second kind are imposed on the artificial boundary (where the field is zero). This idea allows one to extend the class of problems which can be solved by analytical methods. Using the TREE method the solution is constructed by means of the method of separation of variables. However, some steps of the solution procedure require the use of numerical methods. In particular, one of the steps in the TREE method is the calculation of complex eigenvalues. The eigenvalue problem reduces to the solution of the equation

$$
\varphi(z)=0
$$

where $z$ is a complex which occurs in eddy current testing problems where the boundary conditions between media with different properties are used.

There are two important aspects of the solution of (1). First, no good initial guess for the roots of (1) is known. Second, relatively large number of eigenvalues needs to be computed since the change in impedance of an eddy current coil is represented by an eigenfunction expansion. The numerical procedure used to compute the roots of (1) is described in the presented paper. Preliminary results leading to this publication were reported at the international conference in Šibenik, Croatia, in 2013 [6].

\section{Three Types of EdDy CURRENT Testing Problem}

In this section we describe three models where the solution of (1) represents an important computational step. The geometry of the first model is shown in Fig. 1.

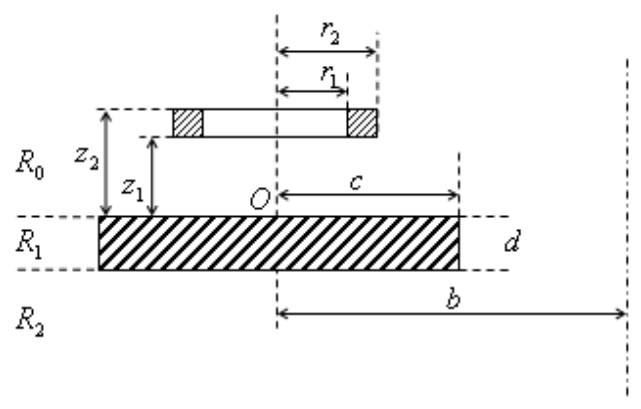

Fig. 1. Coil with alternating current above the conducting cylinder.

This model can be used in practice to analyze objects inserted in coin validators. Consider a coil with alternating current of frequency $\omega$ located above the conducting cylinder. The inner and outer radii of the coil are $r_{1}$ and $r_{2}$, respectively. The coil is located at a distance $z_{1}$ from the conducting cylinder. The height of the coil is $z_{2}-z_{1}$. The parameters describing the cylinder are as follows: $c$ is the radius, $d$ is the height and $\sigma$ is the electrical conductivity (the cylinder is non-magnetic). We used the TREE method [3] to solve the problem. It was assumed that the electromagnetic 
$2014 / 53$

field is exactly zero at a sufficiently large radial distance $b$ from the axis of the coil.

The problem was solved by method of separation of variables where the solution is expressed in terms of the vector potential. The finite size of the cylinder imposes some additional conditions. In particular, two sets of eigenvalues have to be calculated. One set of eigenvalues is obtained from the condition that the vector potential in regions $R_{0}, R_{1}$ and $R_{2}$ is equal to zero. The corresponding eigenvalues are given by

$$
\lambda_{i}=\frac{\alpha_{i}}{b}, i=1,2, . ., n
$$

where $\alpha_{i}$ are the roots of the equation

$$
J_{1}(\alpha)=0
$$

Here $J_{1}(\alpha)$ is the Bessel function of the first kind of order one. The roots $\alpha_{i}$ can be easily computed with Mathematica. The corresponding Mathematica script is shown in Fig. 2 where the first ten zeros of (3) are shown.

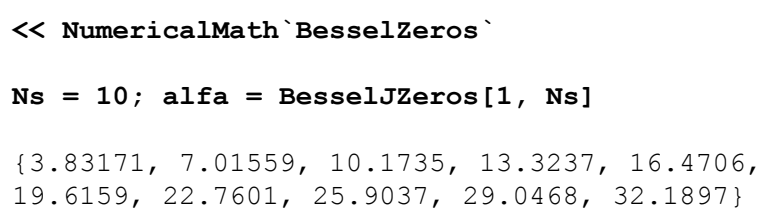

Fig. 2. Mathematical code for the computation of the zeros of (3).

The corresponding eigenvalues $p_{i}$ are the roots of the equation

$$
p J_{1}(q c) T_{1}^{\prime}(p c)-q J_{1}^{\prime}(q c) T_{1}(p c)=0
$$

where

$T_{1}\left(p_{i} r\right)=J_{1}\left(p_{i} r\right) Y_{1}\left(p_{i} b\right)-J_{1}\left(p_{i} b\right) Y_{1}\left(p_{i} r\right)$,

$Y_{i}(x)$ is the Bessel function of the second kind of order one,

$q_{i}=\sqrt{{p_{i}}^{2}-j \omega \sigma \mu_{0}}, j$ is the imaginary unit,

$\omega=2 \pi f$ and $\mu_{0}$ is the magnetic constant.

The second model is shown in Fig. 3.

The model can be used to analyze the effect of corrosion in metal plates. The corresponding eigenvalue relation in this case has the form

$$
p_{i} T_{1}\left(q_{i} c\right) J_{1}^{\prime}\left(p_{i} c\right)=q_{i} T_{1}^{\prime}\left(q_{i} c\right) J_{1}\left(p_{i} c\right)
$$

where $T_{1}\left(q_{i} c\right)=J_{1}\left(q_{i} c\right) Y_{1}\left(q_{i} b\right)-J_{1}\left(q_{i} b\right) Y_{1}\left(q_{i} c\right)$.

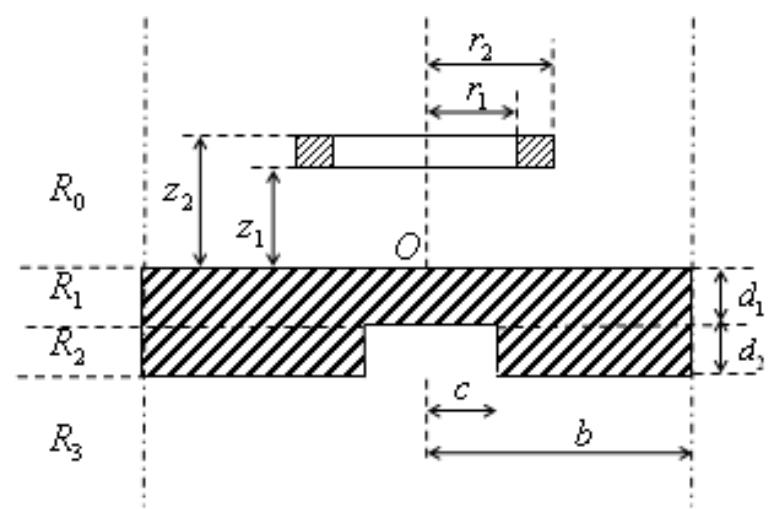

Fig. 3. Coil of finite dimensions above the conducting plate with a cylindrical hole.

The third model is shown in Fig. 4 and can be used to test the quality of spot welding. A cast core (represented by a cylinder of radius $c$ ) is formed during the welding process. The conductivity $\sigma_{2}$ of the cylinder is (as experimental data show) close to the conductivity $\sigma_{1}$ of the surrounding medium (however, no restriction on $\sigma_{2}$ is imposed in our analysis). The eigenvalue relation is given by (5), where $q_{i}=\sqrt{p_{i}^{2}-j \omega \sigma_{2} \mu_{0}}$.

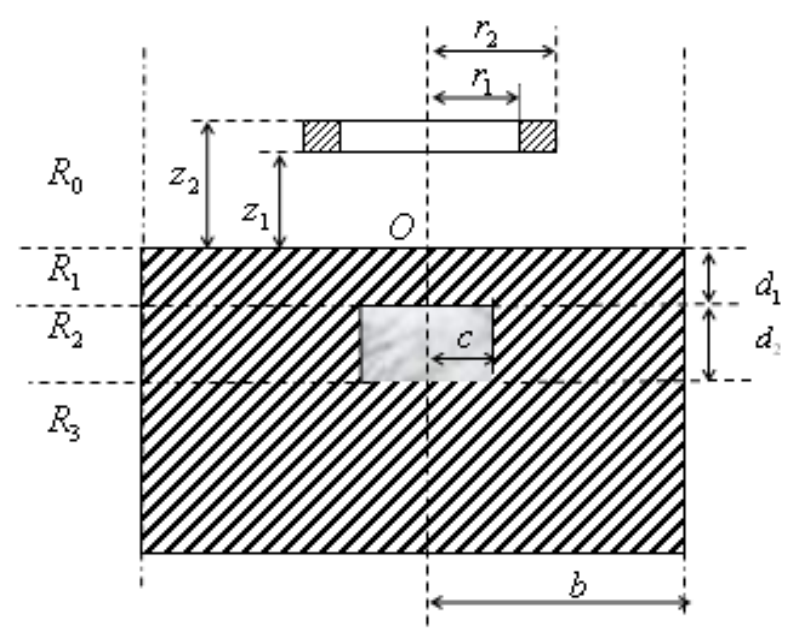

Fig. 4. Coil with finite dimensions above the half-space with a flaw.

\section{NUMERICAL PROCEDURE}

The solution of eigenvalue problems (4) and (5) is based on the idea described in [7] and [8]. It is known from the theory of complex variables that the number of zeros $n_{0}$, of an analytic function $\varphi(z)$ inside a closed contour $C$ is equal to

$$
n_{0}=\frac{1}{2 \pi i} \oint_{C} \frac{\varphi^{\prime}(z)}{\varphi(z)} d z .
$$


In addition, it can be shown [7], [8] that

$$
z_{n}=\frac{1}{2 \pi i} \oint_{C} z^{n} \frac{\varphi^{\prime}(z)}{\varphi(z)} d z=\sum_{i=1}^{k} \zeta_{i}^{n}
$$

where $\zeta_{i}, i=1,2, . ., k$ are zeroes (simple or multiple) of $\varphi(z)$ inside $C$ and $n=1,2, . ., k$.

It is suggested in [7] and [8] that using (7) one can construct a polynomial of degree $k$ roots of which are the same as the roots of $\varphi(z)$. The roots of the polynomial (and, therefore, the roots of $\varphi(z)$ ) can be easily computed (for example, Mathematica command Roots can be used). However, the number $k$ should not be too large since roots of a polynomial of high degree can be quite sensitive to the coefficients [9]. We developed an algorithm for the computation of roots of (1) for the case where there are at most two eigenvalues inside $C$. The contour $C$ is a rectangle (which can easily be sub-divided into smaller rectangles, if necessary). If there are two eigenvalues inside $C$ then the program divides the rectangle by smaller rectangles until there is only one root of (1) inside $C$. The eigenvalue is computed by formula (7) with $n=1$. As an example we present here computational results for the first problem (detailed calculations for other cases can be found in [10]). The program for the computation of complex eigenvalues can also be found in [10].

The first 5 roots of (3) are shown in Fig. 5 for the following parameters of the problem $\sigma=9.6 \mathrm{Ms} / \mathrm{m}, c=19.75 / 2 \mathrm{~mm}$, $b=60 \mathrm{~mm}, \omega=1 \mathrm{kHz}$.

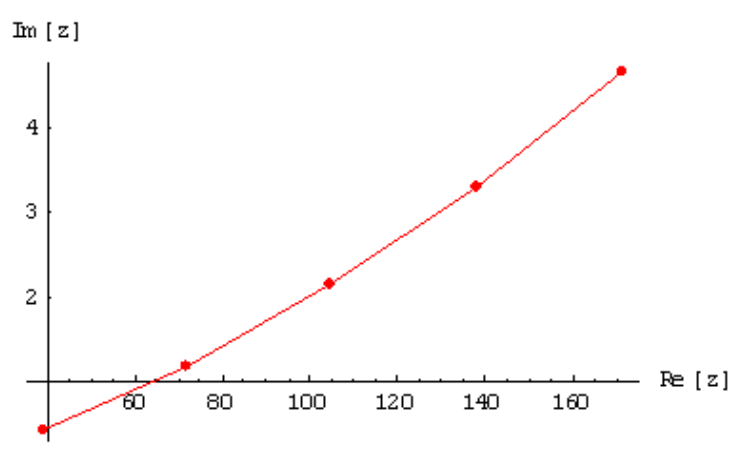

Fig. 5. First five roots of (3).

\section{CONCLUSION AND DIRECTION OF FUTURE WORK}

An algorithm for the computation of complex eigenvalues for eddy current testing problems is described in the paper. The algorithm is implemented in Mathematica. Calculations show that the method is reliable and efficient (at least, for relatively low frequencies in the range from $1 \mathrm{kHz}$ to $10 \mathrm{kHz}$ ). Determination of complex eigenvalues is one of the important steps in the TREE method (see examples in [11] and [12]). Recently asymmetric problems of eddy current testing problems were solved in [13]-[16]. Solutions were obtained using the second order vector potential formulation. The author is currently working on the application of the TREE method and second order vector potential formulation to asymmetric problems in cylindrical coordinates.

\section{REFERENCES}

[1] J. A. Tegopoulos and E. E. Kriezis, Eddy currents in linear conducting media. Amsterdam: Elsevier, 1985.

[2] M. Ya. Antimirov, A. A. Kolyshkin, and R. Vaillancourt, Mathematical models in eddy current testing. Montreal: CRM, 1997.

[3] T. P. Theodoulidis, and E. E. Kriezis, Eddy current canonical problems (with application to nondestructive evaluation). Duluth: Tech Science, 2006.

[4] N. Ida, Numerical modeling for electromagnetic non-destructive evaluation, Chapman \& Hall, 1995.

[5] T. P. Theodoulidis, and E. E. Kriezis, "Series expansions in eddy current nondestructive evaluation models," Journal of materials processing technology, vol. 161, pp. 343-347, 2005. http://dx.doi.org/10.1016/j.jmatprotec.2004.07.048

[6] V. Koliskina and I. Volodko, "Determination of complex eigenvalues for axisymmetric problems in eddy current testing," $8^{\text {th }}$ conference on applied mathematics and scientific computing, 2013.

[7] L. M. Delves, and J. N. Lynness, "A numerical method for locating the zeros of an analytic function," Mathematics of Computation, vol. 21, pp. 543-560, 1967. http://dx.doi.org/10.1090/S0025-5718-1967-0228165-4

[8] J. N. Lynness, "Numerical algorithms based on the theory of complex variable," Proc. ACM, pp. 125-133, 1967.

[9] G.E. Forsythe, M.A. Malcolm, and C.B. Moler, Computer methods for mathematical computations, Prentice-Hall: Englewood Cliffs, 1977.

[10] V. Koliskina, "Analytical and quasi-analytical solutions of direct problems in eddy current testing," $\mathrm{PhD}$ thesis, Riga Technical University, Latvia, 2014.

[11] H. Sun, J.R. Bowler, and T.P. Theodoulidis, "Eddy currents iinduced in a finite length layered rod by a coaxial coil," IEEE Transactions on Magnetics, vol. 41, no. 9, 2005, pp. 2455-2461. http://dx.doi.org/10.1109/TMAG.2005.855439

[12] T. Theodoulidis, and J. R. Bowler, "Eddy current interaction of a long coil with a slot in a conductive plate," IEEE Transactions on Magnetics, vol. 41, no. 4, 2005, pp. 1238-1247. http://dx.doi.org/10.1109/TMAG.2005.844838

[13] A. Scarlatos, and T. "Theodoulidis, Impedance calculation of a bobbin coil in a conductive tube with eccentric walls," IEEE Transactions on Magnetics, vol. 46, no. 11, 2010, pp. 3885-3892. http://dx.doi.org/10.1109/TMAG.2010.2064331

[14] T. Theodoulidis, and J. R. Bowler, "Interaction of an eddy current coil with a right-angled conductive wedge," IEEE Transactions on Magnetics, vol. 46, no. 4, 2010, pp. 1034-1042. http://dx.doi.org/10.1109/TMAG.2009.2036724

[15] A. Scarlatos, and T. Theodoulidis, "Analytical treatment of eddy-current induction in a conducting half-space with a cylindrical hole parallel to the surface," IEEE Transactions on Magnetics, vol. 47, no. 11, 2011, pp. 4592-4599. http://dx.doi.org/10.1109/TMAG.2011.2158550

[16] A. Scarlatos, and T. Theodoulidis, "Solution to the eddy-current induction problem in a conducting half-space with a vertical cylindrical borehole," Proceedings of the Royal Society A: Mathematical, Physical and Engineering Sciences, vol. 468(2142), 2012, pp. 1758-1777. http://dx.doi.org/10.1098/rspa.2011.0684

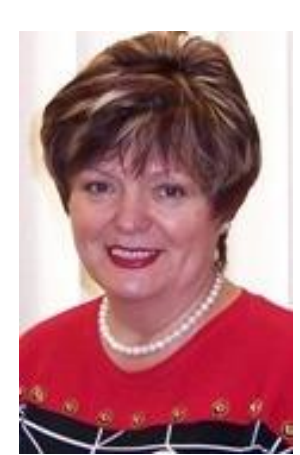

Valentina Koliskina defended her Doctoral Thesis "Aanlytical and quasi-analytical solutions of direct problems in eddy current testing" in the University of Latvia, Riga, Latvia in 2014. Presently she is a lecturer with the Department of Engineering Mathematics of Riga Technical University, Latvia. Her research interests are: analytical and semi-analytical solutions of direct problems in eddy current testing. Since 2007 she has participated in more than 20 international conferences. She is the author of more than 30 papers published in peer-reviewed journals and conference proceedings.

E-mail: valentina.koliskina@ rtu.lv 